\title{
Histopathological Findings of Cystic Endometrial Morphology Based on Ultrasonographic Imaging in Premenopausal Women
}

\author{
Tugba KINAY', Metin KAPLAN ${ }^{1}$, Fulya KAYIKCIOGLU ${ }^{1}$, Jale KARAKAYA² \\ Ankara, Turkey
}

\begin{abstract}
OBJECTIVE: To evaluate the histopathological findings of cystic endometrial morphology based on ultrasonographic imaging in premenopausal women.

STUDY DESIGN: The medical records of 3607 premenopausal women that underwent an ultrasonographic examination at a tertiary care center were reviewed, as were endometrial biopsy findings in 816 of the women. These 816 women were divided into 2 groups according to ultrasonographic endometrial findings: the cystic group and the non-cystic group. Clinical and histopathological findings in the 2 groups were compared. Statistical analysis was performed using independent samples $t$ - test, chisquare test, and binary logistic regression analysis.
\end{abstract}

RESULTS: Three hundred and eightyone (46.7\%) of the women that underwent endometrial biopsy had cystic endometrium, whereas $435(53.3 \%)$ had non-cystic endometrium. The most common histopathological finding in the cystic group was endometrial hyperplasia without atypia (44.6\%). Cystic morphology was nearly 8-fold more common in the women with endometrial hyperplasia without atypia (95\% $\mathrm{Cl}$ : 5.43 11.67). The premalignant and malignant pathology rates in the cystic group and non-cystic group were similar $(1.1 \%$ vs. $0.5 \%[p=0.426]$ and $0.8 \%$ vs. $0.5 \%[p=0.669]$, respectively). Secretory endometrium was less common in the cystic group than in the non-cystic group (5.0\% vs. $37.9 \%[p<0.001])$.

CONCLUSION: Cystic endometrial morphology based on ultrasonographic imaging was more common in women with endometrial hyperplasia without atypia. There wasn't an association between cystic endometrium and other endometrial pathologies or functional endometrium.

Keywords: Cystic endometrium, Endometrium, Endometrial hyperplasia, Premenopausal, Ultrasonography

\section{Gynecol Obstet Reprod Med 2019;25(1):33-37}

${ }^{1}$ University of Health Sciences, Etlik Zubeyde Hanim Women's Health Training and Research Hospital, Ankara

${ }^{2}$ Hacettepe University, Faculty of Medicine Department of Biostatistics, Ankara

Address of Correspondence: Tugba Kinay

Department of Obstetrics and Gynecology, University of Health Sciences, Etlik Zubeyde Hanim Women's Health Training and Research Hospital 06010 Kecioren, Ankara, Turkey tkinay@hotmail.com

Submitted for Publication: 08.01 .2018

Accepted for Publication:

ORCID IDs of the authors:

T.K.: 0000-0001-5340-1025, M.K.: 0000-0001-8321-0262,

F.K.: 0000-0002-1078-0982, J.K.: 0000-0002-7222-7875

\begin{tabular}{|c|c|}
\hline Quick Response Code: & Access this article online \\
\cline { 2 - 2 } & Website: www.gorm.com.tr \\
\hline & e- mail: info@gorm.com.tr \\
\hline
\end{tabular}

How to cite this article: Kinay T. Kaplan M. Kayikcioglu F. Karakaya J. Histopathological Findings of Cystic Endometrial Morphology Based on Ultrasonographic Imaging in Premenopausal Women. Gynecol Obstet Reprod Med 2019;25(1):33-37

\section{Introduction}

Curettage and hysteroscopy guided endometrial biopsy are the most reliable diagnostic tools for endometrial pathologies $(1,2,3)$; however, clinicians have been urged to find alternative methods for evaluating the endometrium because of these methods are expensive, uncomfortable, and invasive procedures. Measurement of endometrial thickness via ultrasonography is an easy and tolerable method that has become the firstline test for evaluating abnormal uterine bleeding. Endometrial thickness can be used to predict malignancy in postmenopausal women. Endometrial carcinoma is unlikely to occur in women with postmenopausal bleeding and endometrial thickness $<4-5$ $\mathrm{mm}$ (4-6); however, measurement of endometrial thickness via ultrasonography has limited value in premenopausal women. Studies on the association between endometrial thickness and endometrial pathology in premenopausal women have reported inconsistent findings (7-10). Ozdemir et al. (7) reported that endometrial pathologies are more common in premenopausal women with abnormal uterine bleeding and endometrial thickness $>8 \mathrm{~mm}$; however, other studies indicate 
that endometrial thickness has a little predictive value for endometrial pathologies in premenopausal women (8-11). These earlier conflicting findings show that investigation of the association between ultrasonographic parameters other than the endometrial thickness and endometrial pathology in premenopausal women is required.

Ultrasonographic endometrial morphology is also useful for assessing endometrial pathology. Recent studies show that evaluation of endometrial thickness and morphology together improves the accuracy of transvaginal ultrasonography for the diagnosis of endometrial carcinoma in postmenopausal women (12-15). In these studies, numerous ultrasonographic morphological characteristics have been examined together, and heterogeneous endometrial echogenicity, intrauterine fluid, and focal lesions with heterogeneous echogenicity and/or an irregular surface were more common in malignant cases than in non-malignant cases $(13,14)$. In addition, a few studies investigated the association between multiple ultrasonographic endometrial morphologies and endometrial pathologies in premenopausal women $(9,16)$. The present study aimed to determine the histopathological findings of cystic endometrial morphology based on ultrasonographic imaging in premenopausal women.

\section{Material and Method}

The medical records of 3607 premenopausal women that underwent transvaginal ultrasonography at gynecology department of a tertiary care center between April 2012 and January 2014 were retrospectively reviewed. Women underwent endometrial biopsy due to cystic endometrial morphology, endometrial thickness $>15 \mathrm{~mm}$ on any day of of menstrual cycle, and abnormal uterine bleeding were included the study. Women with endometrial thickness $\leq 14 \mathrm{~mm}$, obvious intracavitary focal lesions, such as an endometrial polyp or submucosal leiomyoma, tamoxifen using postmenopausal women, and those that had not undergone endometrial biopsy were excluded. Regular or irregular heavy menstrual bleeding and intermenstrual bleeding were defined as abnormal uterine bleeding. Longer than 8 days and/or more than $80 \mathrm{ml}$ bleeding were considered heavy menstrual bleeding. Bleeding between the cyclic menses was considered intermenstrual bleeding. Based on these criteria, 816 of 3607 women with or without cystic endometrium that underwent endometrial biopsy were included in this retrospective cohort study. Signed informed consent was obtained from all the participants at the time of endometrial biopsy. This study designed as a retrospective data review; thus, ethics committee approval was not required.

Patient clinical data, histopathological findings, and ultrasonographic findings were obtained from the hospital's computer database. Data was acquired from the examinations performed on any day of the menstrual cycle by a sonographer with 15 years of experience on gynecologic ultrasonography.
Sonographic evaluation using a 6-10-MHz Logiq P5 transvaginal probe (GE Healthcare, Inc., Milwaukee, Wisconsin, U.S.A.) in the lithotomic position with an empty bladder included the following steps: endometrial scanning in the longitudinal and transverse planes, evaluation of endometrial echogenicity and cystic areas within the endometrium, and measurement of the thickest part of endometrium in the longitudinal plane or measurement of 2 endometrial walls separately and recording sum of the 2 measurements as endometrial thickness in the presence of intracavitary fluid.

The participants were divided into 2 groups according to the presence of cystic areas within the endometrium, as shown in figure 1a,b: cystic group: cystic areas within the endometrium; non-cystic group: cystic areas were absent and only endometrial homogeneous echogenicity was observed. Endometrial biopsy using an outpatient endometrial sampling device was performed within 3 days of ultrasonographic examination. Histopathological findings in the 2 groups were compared.

Statistical analysis was performed using IBM SPSS Statistics for Windows v.21.0 (IBM Corp., Armonk, NY). Continuous and categorical variables are presented as mean \pm $\mathrm{SD}$, and number and percentage. Differences in continuous data between the 2 groups were compared using the independent samples $t$ - test. The chi-square test was used to analyze differences in categorical variables between the 2 groups. The odds ratio (OR) and 95\% confidence interval (CI) of OR were calculated using binary logistic regression analysis. The level of statistical significance was set at $p<0.05$.

\section{Results}

Among 3607 premenopausal women, 816 met the study's inclusion criteria and underwent endometrial biopsy. Based on ultrasonographic imaging, 381 (46.7\%) women had heterogeneous endometrial echogenicity and cystic areas within the endometrium (cystic group) and 435 (53.3\%) had homogenous endometrial echogenicity (non-cystic group). Demographic, clinical, and ultrasonographic data are shown in table 1. Mean age in the cystic and non-cystic groups was similar (43.6 \pm 6.1 years and $44.3 \pm 6.7$ years, respectively $[p=0.154]$ ). Mean endometrial thickness was higher in the cystic group than in the non-cystic group $(22.7 \pm 4.3 \mathrm{~mm}$ vs. $15.6 \pm 1.7 \mathrm{~mm}$, respectively $[p<0.001])$. Among the study participants, 601 (73.7\%) had abnormal uterine bleeding, and 215 (26.3\%) had other symptoms, including pelvic pain and secondary amenorrhea, or were asymptomatic. The abnormal uterine bleeding rate was significantly higher in the cystic group than in the non-cystic group (78.7\% [300/381] vs. $69.2 \%[301 / 435][p=0.002])$.

Histopathological findings in the cystic and non-cystic groups are shown in table 2. Cystic endometrial morphology based on ultrasonographic imaging was frequently associated with benign endometrial pathologies, of which the most common was endometrial hyperplasia without atypia (44.6\% 
[170/381]). Cystic endometrial morphology was 7.96-fold more common than non-cystic morphology in the women with endometrial hyperplasia without atypia (95\% CI: 5.43-11.67). In the non-cystic group the most common histopathological finding was functional endometrium (65.5\% [285/435]). In all, $165(37.9 \%)$ women had secretory endometrium and 120 $(27.6 \%)$ had proliferative endometrium. The rates of premalignant and malignant pathologies in the 2 groups were similar (endometrial hyperplasia with atypia: 1.1\% [4/381] in the cystic group vs. $0.5 \%$ [2/435] in the non-cystic group [ $p=$
$0.426]$; endometrial carcinoma: $0.8 \%$ [3/381] in the cystic group vs. $0.5 \%$ [2/435] in the non-cystic group $[p=0.669])$.

The cystic and non-cystic endometrial morphology rates for each histopathological finding are shown in figure 2. In total, $81 \%(170 / 210)$ of the women with histopathological findings of endometrial hyperplasia without atypia had cystic endometrium based on ultrasonography. In contrast, just $10.3 \%(19 / 184)$ of women with secretory endometrium based on histopathology had cystic endometrial morphology based on ultrasonographic imaging.
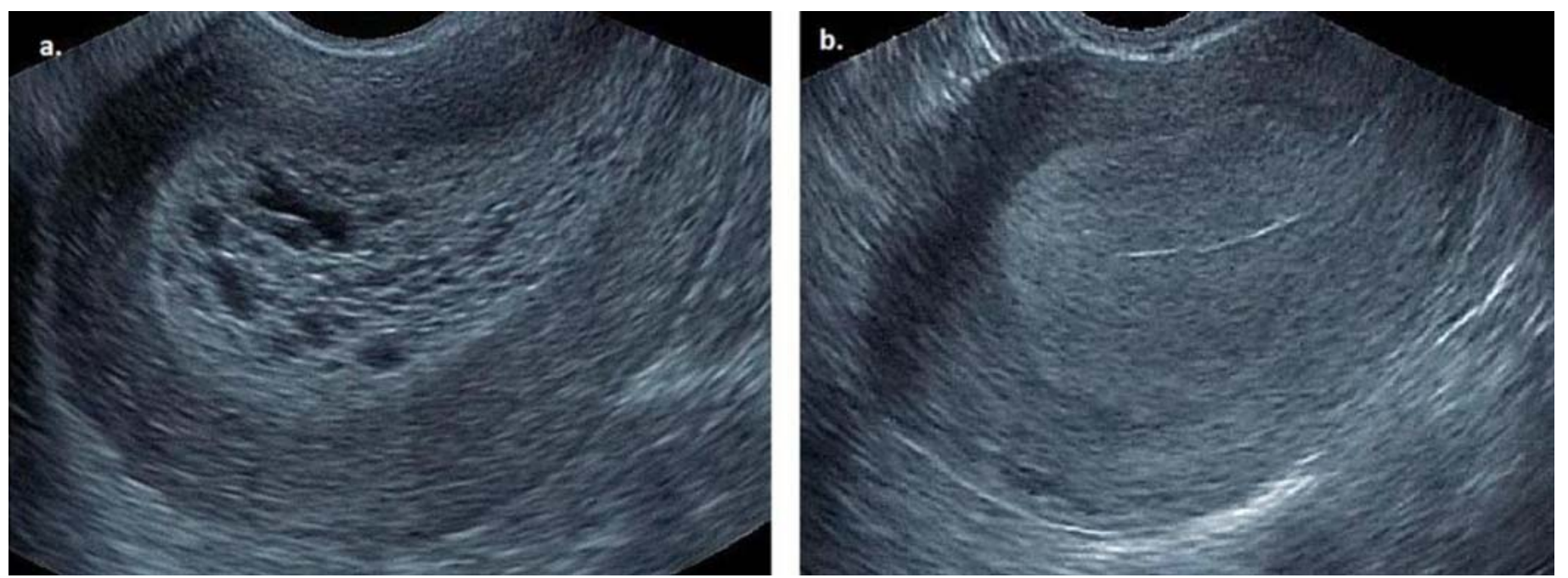

Figure 1a: Cystic endometrial morphology. Cystic areas are seen in the heterogeneous endometrium. b: Non-cystic endometrial morphology. Endometrial echogenicity is homogenous.

Table 1: Demographic, clinical, and ultrasonographic findings in the cystic and non-cystic groups

\begin{tabular}{|c|c|c|c|}
\hline & $\begin{array}{l}\text { Cystic } \\
n=381\end{array}$ & $\begin{array}{c}\text { Non-Cystic } \\
n=435\end{array}$ & $p$ \\
\hline Age (years) & $43.6 \pm 6.1$ & $44.3 \pm 6.7$ & 0.154 \\
\hline Abnormal uterine bleeding, $\mathrm{n}(\%)$ & $300(78.7)$ & $301(69.2)$ & 0.002 \\
\hline Endometrial thickness $(\mathrm{mm})$ & $22.7 \pm 4.3$ & $15.6 \pm 1.7$ & $<0.001$ \\
\hline \multicolumn{4}{|l|}{ Menstrual cycle phase } \\
\hline Proliferative phase, $\mathrm{n}(\%)$ & $105(27.6)$ & $129(29.7)$ & 0.509 \\
\hline Secretory phase, $n(\%)$ & $94(24.7)$ & $178(40.9)$ & $<0.001$ \\
\hline Menses, $\mathrm{n}(\%)$ & $182(47.7)$ & $128(29.4)$ & $<0.001$ \\
\hline
\end{tabular}

Table 2: Histopathological findings in the cystic and non-cystic groups

\begin{tabular}{|c|c|c|c|c|}
\hline Histopathological Findings & $\begin{array}{l}\text { Cystic } \\
n=381\end{array}$ & $\begin{array}{c}\text { Non-cystic } \\
n=435\end{array}$ & Odds ratio $(95 \% \mathrm{Cl})$ & $p$ \\
\hline Benign endometrium & $\mathrm{n}(\%)$ & $\mathrm{n}(\%)$ & & \\
\hline Endometrial polyp & $95(24.9)$ & $106(24.4)$ & $1.03(0.75-1.42)$ & 0.851 \\
\hline Endometrial hyperplasia without atypia & $170(44.6)$ & $40(9.2)$ & $7.96(5.43-11.67)$ & $<0.001$ \\
\hline \multicolumn{5}{|l|}{ Premalignant and malignant endometrium } \\
\hline Endometrial hyperplasia with atypia & $4(1.1)$ & $2(0.5)$ & $2.29(0.42-12.61)$ & 0.426 \\
\hline Endometrial carcinoma & $3(0.8)$ & $2(0.5)$ & $1.72(0.29-10.34)$ & 0.669 \\
\hline \multicolumn{5}{|l|}{ Functional endometrium } \\
\hline Proliferative endometrium & $90(23.6)$ & $120(27.6)$ & $0.81(0.59-1.11)$ & 0.186 \\
\hline Secretory endometrium & $19(5.0)$ & $165(37.9)$ & $0.09(0.05-0.14)$ & $<0.001$ \\
\hline
\end{tabular}




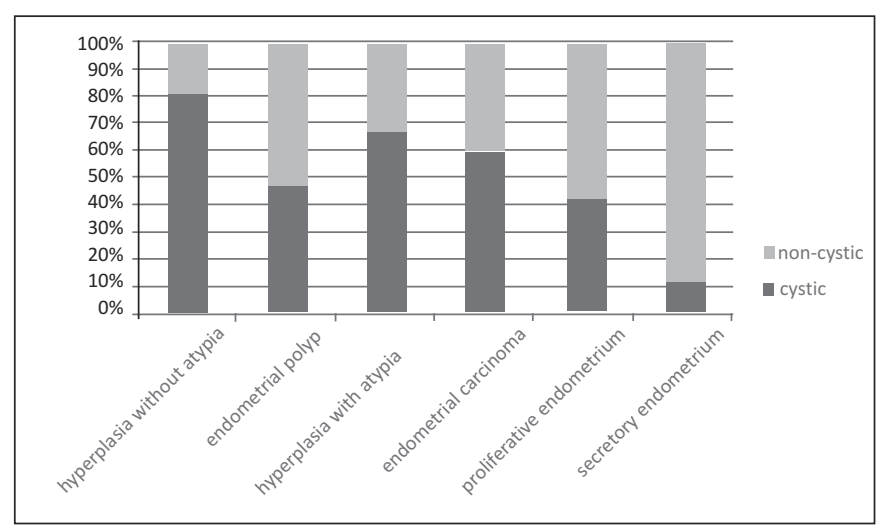

Figure 2: Distribution of cystic and non-cystic endometrial morphologies for each histopathological finding. The cystic endometrial morphology rate was significantly higher than the non-cystic morphology rate in women with endometrial hyperplasia without atypia, and was significantly lower than the noncystic morphology rate in women with secretory endometrium.

\section{Discussion}

Histopathological examination is the gold standard for diagnosing endometrial pathologies (1); however, specimen collection requires expensive invasive procedures, such as dilatation and curettage, and hysteroscopy; therefore, transvaginal ultrasonography, a widely available noninvasive, safe, and cost-effective procedure has become the first line test in clinical practice for evaluating abnormal uterine bleeding. The predictive value of transvaginal ultrasonographic findings for the diagnosis of endometrial pathologies has been investigated in numerous studies. Ultrasonographic measurement of endometrial thickness and evaluation of the vascular pattern in the endometrial cavity are useful for differentiating benign and malign endometrial pathologies (4-16). The present study also shows that ultrasonographic assessment of endometrial morphology is useful for diagnosing endometrial pathologies.

The present study's findings show that endometrial cysts observed ultrasonographically in premenopausal women are generally associated with endometrial hyperplasia without atypia, and that the ability of this ultrasonographic finding to predict other endometrial pathologies is low. In the present study the rates of cystic and non-cystic endometrial morphology did not differ significantly in women with endometrial polyp, endometrial hyperplasia with atypia, and endometrial carcinoma. Cystic endometrial morphology based on ultrasonography was also noted in women with functional endometrium, especially proliferative endometrium. Cystic endometrium was ultrasonographically observed in $42.9 \%$ of women with proliferative endometrium. On the other hand, the rate of cystic endometrial morphology was significantly lower in women with secretory endometrium.

During the proliferative phase of menstrual cycle progressive mitotic growth of the decidua functionalis occurs, and endometrial glands that are initially straight, narrow, and short subsequently become longer and tortuous. Endometrial stroma is observed as a dense compact layer during this phase of men- strual cycle. Then, progesterone secretion increases, shifts the histological appearance of the endometrium to the secretory phase. During the secretory phase, the proliferation of glandular epithelium stops and stromal edema progressively increases (17). Diffuse stromal edema could lead to homogenous endometrial echogenicity, which might be the cause of the significant lower rate of cystic endometrial morphology on ultrasonographic examination in the study cohort. In contrast, dilated or cystic glands and an increase in the glandular-to-stromal ratio without cytological atypia are observed in histopathological examination of endometrial hyperplasia without atypia (17). Enlarged glands and decreased stromal tissue could cause the endometrium to ultrasonographically appear cystic in patients with endometrial hyperplasia without atypia.

Only a few studies have investigated the association between ultrasonographically based cystic endometrial morphology and intracavitary pathologies. Earlier studies that included postmenopausal women showed that cystic endometrial morphology has low specificity for the diagnosis of endometrial carcinoma $(14,15,18)$. Opolskiene et al. (18) studied 120 patients with postmenopausal bleeding and endometrial thickness $\geq 4.5 \mathrm{~mm}$, reporting that cystic endometrium had low sensitivity and specificity ( $23 \%$ and $42 \%$ respectively) for the diagnosis of endometrial carcinoma. The present findings show that the risk of endometrial carcinoma was low in premenopausal women with cystic endometrial morphology; only $0.8 \%$ of women with cystic endometrium had endometrial carcinoma, which is similar to the rate $(0.5 \%)$ in women with non-cystic endometrium.

Randelzhofer et al. reported that a homogeneous cystic endometrial structure is commonly correlated with endometrial hyperplasia and endometrial polyps (12). The present findings show that there was a significant association between endometrial hyperplasia without atypia and cystic endometrial morphology in premenopausal women; however, cystic endometrium was not predictive of endometrial polyps. The differences in findings might be related to differences in the 2 study's cohorts; postmenopausal women and premenopausal women with obvious endometrial polyps were not included in the present study.

There are few studies on the association between ultrasonographically based cystic endometrial morphology and intracavitary pathologies in premenopausal women $(9,16)$. Kim et al. analyzed ultrasonographic findings and endometrial biopsy results in 162 premenopausal and perimenopausal women, reporting that endometrial stripe anomalies were predictive of endometrial hyperplasia and carcinoma (9). Irregular, cystic, and heterogeneous polypoid masses, abnormally distended endometrium, and intracavitary fluid were defined as endometrial stripe anomalies, and the relationship between endometrial pathologies and all of these anomalies were analyzed together; however, the histopathological findings of women with cystic endometrium only were not reported in their study. Another study that included premenopausal and 
postmenopausal patients did not note an association between endometrial carcinoma and cystic endometrium based on ultrasonography (16). Cystic endometrial morphology was noted in 21 of the 350 women $(6 \%)$ included in the study. The most common histopathological finding was proliferative endometrium (57.1\%), and none of the patients with cystic endometrium had endometrial carcinoma. Endometrial hyperplasia occurred in $14.3 \%$ of patients with cystic endometrium. In the present study, endometrial hyperplasia without atypia rate was $44.6 \%$ in premenopausal women with cystic endometrial morphology.

Unlike earlier studies that investigated multiple morphological findings, the present study analyzed only ultrasonographically based endometrial morphology. To the best of our knowledge, the present study is the largest to analyze the cystic endometrial structure. The major limitation of the present study is its retrospective design. Even though data were reviewed retrospectively, all ultrasonographic examinations were performed by the same experienced sonographer; thus, individual differences that can occur during the evaluation of endometrial morphology were eliminated.

In conclusion, the present findings show that the examination of endometrial morphology via transvaginal ultrasonography can aid the diagnosis of endometrial pathologies. In the present study ultrasonographically based cystic endometrial morphology was more common in premenopausal women with benign endometrial pathologies than in those with premalignant/malignant pathologies. The most common pathology was endometrial hyperplasia without atypia in those with cystic endometrial morphology. The endometrial polyp rate was similar in the women with and without cystic endometrial morphology.

: Confict of Interest: There are no conflicts of interest in connection with this paper.

\section{References}

1. Grimes DA. Diagnostic dilation and curettage: a reappraisal. Am J Obstet Gynecol. 1982;142(1):1-6.

2. Su H, Huang L, Huang KG, Yen CF, Han CM, Lee CL. Accuracy of hysteroscopic biopsy, compared to dilation and curettage, as a predictor of final pathology in patients with endometrial cancer. Taiwan J Obstet Gynecol. 2015;54(6): 757-60.

3. Spadoto-Dias D, Bueloni-Dias FN, Elias LV, Leite NJ, Modotti WP, Lasmar RB, et al. The value of hysteroscopic biopsy in the diagnosis of endometrial polyps. Womens Health. 2016;12(4):412-9.

4. Ferrazzi E, Torri V, Trio D, Zannoni E, Filiberto S, Dordoni D. Sonographic endometrial thickness: a useful test to predict atrophy in patients with postmenopausal bleeding. An Italian multicenter study. Ultrasound Obstet Gynecol. 1996;7(5): 315-21.

5. Van den Bosch T, Van Schoubroeck D, Domali E, Vergote I, Moerman P, Amant F, et al. A thin and regular endometrium on ultrasound is very unlikely in patients with endometrial malignancy. Ultrasound Obstet Gynecol. 2007;29(6):674-9.

6. Gull B, Karlsson B, Milsom I, Granberg S. Can ultrasound replace dilation and curettage? A longitudinal evaluation of postmenopausal bleeding and transvaginal sonographic measurement of the endometrium as predictors of endometrial cancer. Am J Obstet Gynecol. 2003;188(2):401-8.

7. Ozdemir S, Celik C, Gezginc K, Kiresi D, Esen H. Evaluation of endometrial thickness with transvaginal ultrasonography and histopathology in premenopausal women with abnormal vaginal bleeding. Arch Gynecol Obstet. 2010;282(4):395-9.

8. Breitkopf DM, Frederickson RA, Snyder RR. Detection of benign endometrial masses by endometrial stripe measurement in premenopausal women. Obstet Gynecol. 2004;104 (1):120-5

9. Kim MJ, Kim JJ, Kim SM. Endometrial evaluation with transvaginal ultrasonography for the screening of endometrial hyperplasia or cancer in premenopausal and perimenopausal women. Obstet Gynecol Sci. 2016;59(3):192-200.

10. Van den Bosch T, Ameye L, Van Schoubroeck D, Bourne T, Timmerman D. Intra-cavitary uterine pathology in women with abnormal uterine bleeding: a prospective study of 1220 women. Facts Views Vis Obgyn. 2015;7(1):17-24.

11. Dreisler E, Sorensen SS, Ibsen PH, Lose G. Value of endometrial thickness measurement for diagnosing focal intrauterine pathology in women without abnormal uterine bleeding. Ultrasound Obstet Gynecol. 2009;33(3):344-8.

12. Randelzhofer B, Prömpeler HJ, Sauerbrei W, Madjar H, Emons G. Value of sonomorphological criteria of the endometrium in women with postmenopausal bleeding: a multivariate analysis. Ultrasound Obstet Gynecol. 2002;19 (1): 62-8.

13. Epstein E, Skoog L, Isberg PE, De Smet F, De Moor B, Olofsson PA, et al. An algorithm including results of grayscale and power Doppler ultrasound examination to predict endometrial malignancy in women with postmenopausal bleeding. Ultrasound Obstet Gynecol. 2002;20(4):370-6.

14. Epstein E, Valentin L. Gray-scale ultrasound morphology in the presence or absence of intrauterine fluid and vascularity as assessed by color Doppler for discrimination between benign and malignant endometrium in women with postmenopausal bleeding. Ultrasound Obstet Gynecol. 2006; 28(1):89-95.

15. Dueholm M, Møller C, Rydbjerg S, Hansen ES, Ørtoft G. An ultrasound algorithm for identification of endometrial cancer. Ultrasound Obstet Gynecol. 2014;43(5):557-68.

16. Ozer A, Ozer S, Kanat-Pektas M. Correlation between transvaginal ultrasound measured endometrial thickness and histopathological findings in Turkish women with abnormal uterine bleeding. J Obstet Gynaecol Res. 2016;42(5):573-8.

17. Olive DL, Palter SV. Reproductive Physiology. In: Berek JS, editor. Berek \& Novak's Gynecology. Philadelphia, USA: Lippincott Williams \& Wilkins 2012:138-159.

18. Opolskiene G, Sladkevicius P, Valentin L. Ultrasound assessment of endometrial morphology and vascularity to predict endometrial malignancy in women with postmenopausal bleeding and sonographic endometrial thickness $>$ or $=4.5 \mathrm{~mm}$. Ultrasound Obstet Gynecol. 2007;30(3):332-40. 